\title{
AVOIDING SELF-REFERENTIAL STATEMENTS
}

\author{
C. SMORYŃSKI
}

\begin{abstract}
Recursion-theoretic proofs of metamathematical results tend to rely on a pair of effectively inseparable r.e. sets and its properties. We establish a special property for a small configuration of such pairs and derive from it some metamathematical results not previously accessible to recursion-theoretic techniques.
\end{abstract}

0. Introduction. The applications of the dual completeness of a pair of effectively inseparable r.e. sets to metamathematical questions are manifold. Since Shepherdson 1960, however, more powerful results have been obtainable by diagonalization within a given theory. In this note, we prove a generalization of Smullyan's dual completeness result (cf. Rogers 1967, Exercise 11.29) and list some metamathematical corollaries not previously obtainable recursion-theoretically.

We let $[e]$ denote the partial recursive function with index $e$, and $W_{e}$ the r.e. set with index $e$. Texy is Kleene's $T$-predicate and, for any assertions, $\exists v R v$, $\exists v S v$, with $R, S$ recursive, we write

$$
\begin{aligned}
& \left.\exists v R v \preccurlyeq \exists v S v: \exists v\left[R v \wedge \forall v^{\prime}<v\right\urcorner S v^{\prime}\right], \\
& \exists v R v \prec \exists v S v: \exists v\left[R v \wedge \forall v^{\prime} \leqslant \neg S v^{\prime}\right] .
\end{aligned}
$$

A disjunction $\exists v T v \vee \exists v U v$ in one of these contexts is assumed rewritten $\exists v(T v \vee U v)$. For r.e. sets $X, Y$, we define

$$
X \preccurlyeq Y:\{x: x \in X \preccurlyeq x \in Y\}, \quad X \prec Y:\{x: x \in X \prec x \in Y\},
$$

where $x \in X, x \in Y$ abbreviate $\exists v$ Texv for appropriate $e$. Note that $X \preccurlyeq Y$ and $Y \prec X$ are simply the sets obtained by applying the Reduction Theorem to $X, Y$. (This notation is due to Dave Guaspari.)

1. A double dual completeness theorem. The main result of this note is the following

THEOREM. Let $(A, C),(B, D)$ be pairs of effectively inseparable r.e. sets with $A \subseteq B, C \subseteq D$. There is a recursive function $f$ such that, for all $x$,

$x \in A$ iff $f x \in A$ iff $f x \in B$

$x \in C$ iff $f x \in C$ iff $f x \in D$.

In words, the conclusion of the theorem simply states that the pair $(A, C)$ is uniformly many-one reducible to both pairs $(A, C)$ and $(B, D)$.

Proof. The proof is simple but devious. By Smullyan's dual completeness

Received by the editors September 27, 1977.

AMS (MOS) subject classifications (1970). Primary 02G99; Secondary 02F25, 02F50, $02 \mathrm{G} 05$.

(1) American Mathematical Society 1978 
result, there is a recursive function $g$ such that, for all $i, j$, the function [ $g(i, j)]$ reduces the pair $\left(W_{i} \preccurlyeq W_{j}, W_{j} \prec W_{i}\right)$ to $(A, C)$. Apply Smullyan's Double Recursion Theorem (Rogers 1967, Theorem 11.10) to obtain indices $a, c$ such that, for $f=[g(a, c)]$ and all $x$,

$$
\begin{aligned}
& x \in W_{a} \Leftrightarrow[f x \in D \vee x \in A . \preccurlyeq f x \in B \vee x \in C], \\
& x \in W_{c} \Leftrightarrow[f x \in B \vee x \in C . \prec . f x \in D \vee x \in A] .
\end{aligned}
$$

Obviously, $W_{a}$ and $W_{c}$ are disjoint.

Claim 1. $W_{a}=A \preccurlyeq C=A ; W_{c}=C \prec A=C$.

To see this, observe

$$
\begin{aligned}
x \in W_{a} & \Rightarrow x \in W_{a}-W_{c} \\
& \Rightarrow f x \in A \subseteq B \wedge f x \notin D, \text { since } A \cap D=\varnothing \\
& \Rightarrow x \in A . \preccurlyeq f x \in B \vee x \in C, \text { by definition of } W_{a} \\
& \Rightarrow x \in A .
\end{aligned}
$$

Similarly, $x \in W_{c} \Rightarrow x \in C$. But also,

$$
x \in A \Rightarrow x \in W_{a} \vee x \in W_{c} \Rightarrow x \in W_{a},
$$

since $x \in W_{c}$ yields $x \in C$ which is disjoint from $A$. Similarly $x \in C \Rightarrow x \in$ $W_{\epsilon}$.

Claim 2. For all $x$,

$$
x \in A \Leftrightarrow f x \in A, \quad x \in C \Leftrightarrow f x \in C .
$$

This is trivial since $f=[g(a, c)]$ and $(A, C)=\left(W_{a}, W_{c}\right)=\left(W_{a} \preccurlyeq W_{c}\right.$, $\left.W_{c} \prec W_{a}\right)$.

Claim 3. For all $x$,

$$
x \in A \Leftrightarrow f x \in B, \quad x \in C \Leftrightarrow f x \in D .
$$

The left-to-right implications follow from Claim 2. For the other direction, assume first that $f x \in B$. A glance at the definition of $W_{a}, W_{c}$ reveals that $x \in W_{a}$ or $x \in W_{c}$. The latter yields $f x \in D$, contrary to assumption. Thus $x \in W_{a}=A$. Similarly one shows $f x \in D$ implies $x \in C$. Q.E.D.

Obviously we can compose a reduction of $(X, Y)$ to $(A, C)$ with $f$ to obtain a simultaneous reduction of any pair of disjoint r.e. sets to $(A, C)$ and $(B, D)$. A second corollary, noticed by $\mathrm{J}$. R. Shoenfield, is this: For $A, B, C, D$ as in the Theorem, any set $X$ interpolated between $A$ and $B, A \subseteq X \subseteq B$, has degree at least $0^{\prime}$. [N.B. Without $C$ and $D$, this need not hold: Creative sets can have recursive interpolants.]

2. Some metamathematical applications. We give a few corollaries concerning the metamathematics of r.e. systems of arithmetic (for definiteness: extensions of Robinson's $\mathcal{R}$ ) that were previously obtainable only via selfreferential formulae (cf. Shepherdson 1960, Smoryński A).

Definitions. A formula $\varphi v_{0} \cdots v_{n-1}$ semirepresents a relation $R \subseteq \omega^{n}$ in a theory $\mathcal{T}$ iff, for all $x_{0}, \ldots, x_{n-1}$,

$$
\sigma \vdash \varphi \bar{x}_{0} \cdots \bar{x}_{n-1} \Leftrightarrow R x_{0} \cdots x_{n-1} \text {. }
$$


$\varphi$ dually semirepresents a disjoint pair of relations, $R, S$ iff $\varphi, \neg \varphi$ semirepresent $R, S$, respectively. $\varphi$ represents $R$ iff $\varphi$ dually semirepresents $R$ and its complement. A formula $\varphi v_{0} \cdots v_{n}$ semirepresents (represents) a partial (total) function $f$ iff (i) $\varphi$ semirepresents (represents) the graph of $f$, and (ii) $\varphi$ satisfies a unicity condition, say,

$$
\mathcal{T} \vdash \varphi v_{0} \cdots v_{n-1} v \wedge \varphi v_{0} \cdots v_{n-1} v^{\prime} \rightarrow v=v^{\prime} .
$$

[This is stronger than necessary for most purposes.]

COROllary 1. Let $\mathcal{T}$ be a consistent r.e. extension of $\mathcal{R}$. For any disjoint pair, $R, S$ of $n$-ary r.e. relations, there is a formula $\varphi v_{0} \cdots v_{n-1} \in \Sigma_{1}$ which dually semirepresents $R, S$ in $\mathcal{T}$; and, moreover, $\varphi v_{0} \cdots v_{n-1}$ defines $R$ in the set of natural numbers.

Proof. Obviously we can assume the Theorem proven for $n$-ary relations. Moreover, by Smullyan's Dual Completeness Theorem, we can assume $R, S$ to be effectively inseparable. So let $\psi_{0}, \psi_{1}$ be $\Sigma_{1}$ definitions of $R, S$ and let $A=R, C=S, B=\left\{\left(x_{0}, \ldots, x_{n-1}\right):\right.$ T $\left.\vdash\left(\psi_{0} \preccurlyeq \psi_{1}\right) \bar{x}_{0} \cdots \bar{x}_{n-1}\right\}$, and $D=$ $\left\{\left(x_{0}, \ldots, x_{n-1}\right): \mathcal{T} \vdash \neg\left(\psi_{0} \preccurlyeq \psi_{1}\right) \bar{x}_{0} \cdots \bar{x}_{n-1}\right\}$. Now simply define $\varphi v_{0} \cdots v_{n-1}$ :

$$
\exists v_{0}^{\prime} \cdots v_{n-1}^{\prime}\left[\chi v_{0} \cdots v_{n-1} v_{0}^{\prime} \cdots v_{n-1}^{\prime} \wedge\left(\psi_{0} \preccurlyeq \psi_{1}\right) v_{0}^{\prime} \cdots v_{n-1}^{\prime}\right],
$$

where $\chi \in \Sigma_{1}$ represents the recursive function $f$ of the Theorem. Q.E.D.

The correctness of the semirepresentation of $R$ is the novel feature of this proof. While it comes free with Shepherdson's proof via self-referential formulae, the correctness has either been lacking in recursion-theoretic proofs of Corollary 1 (Ehrenfeucht and Feferman 1960, Putnam and Smullyan 1960), or has resulted in non- $\Sigma_{1}$ semirepresentations (Hájková and Hájek 1972).

COROllaRy 2. The dual semirepresentation $\varphi$ for disjoint $R, S$ can be chosen uniformly in an r.e. sequence, $\mathcal{T}_{0}, \mathcal{T}_{1}, \ldots$, of consistent extensions of $R$.

The proof is as before: Let $B_{i}, D_{i}$ be the sets of tuples provably in, respectively out of, $R \preccurlyeq S$ in $\mathcal{T}_{i}$ and let $B=\cup_{i} B_{i} \preccurlyeq \cup_{i} D_{i}, D=\cup_{i} D_{i} \prec$ $\cup_{i} B_{i}$.

Again, this result was originally quite easily proven by means of formal diagonalization.

COROLlaRY 3. Let $f$ be partial recursive; $\sigma_{0}, \sigma_{1}, \ldots$ an r.e. sequence of consistent extensions of $\mathcal{R}$. There is a formula $\varphi v_{0} \cdots v_{n} \in \Sigma_{1}$ which correctly uniformly semirepresents $f$ in each $\mathcal{T}_{i}$. Moreover, we can assume

$$
\sigma_{i} \vdash \neg \varphi \bar{x}_{0} \cdots \bar{x}_{n-1} \bar{y} \Leftrightarrow \exists z \neq y\left(f x_{0} \cdots x_{n-1}=z\right) .
$$

Again the result is sharper than the original recursion-theoretic result (Ritchie and Young 1968/1969). We omit the proof.

As a final application we have

Corollary 4. Let $\mathcal{T}_{0} \subsetneq \mathcal{T}_{1}$ be consistent r.e. extensions of $\mathcal{R}$ and let 
$R_{0} \subseteq R_{1}$ be n-ary r.e. relations. There is a formula $\varphi$ such that $\varphi$ semirepresents $R_{i}$ in $\mathcal{T}_{i}$.

Proof. We shall cheat slightly. Di Paola 1966 shows that there is a $\psi_{0}$ which semirepresents $R_{0}$ in $\mathcal{T}_{0}$ and $\omega^{n}$ in $\mathcal{T}_{1}$. So let $\psi_{1}$ uniformly semirepresent $R_{1}$ in $\mathcal{T}_{0}, \mathcal{T}_{1}$ and define $\varphi=\psi_{0} \wedge \psi_{1}$. Q.E.D.

Di Paola's full result required there to be a recursive interpolant between $R_{0}$ and $R_{1}$.

\section{REFERENCES}

A. Ehrenfeucht and S. Feferman

1960 Representability of recursively enumerable sets in formal theories, Arch. Math. Logik Grundlagenforsch. 5 37-41.

M. Hájková and P. Hájek

1972 On interpretability in theories containing arithmetic, Fund. Math. 76 131-137.

R. A. di Paola

1966 On sets represented by the same formula in distinct consistent axiomatizable Rosser theories, Pacific J. Math. 18 445-456.

H. Putnam and R. M. Smullyan

1960 Exact separation of recursively enumerable sets within theories, Proc. Amer. Math. Soc. 11 574-577.

R. W. Ritchie and P. R. Young

1968/1969 Strong representability of partial functions in arithmetic theories, Information Sci. 1 189-204.

H. Rogers, Jr.

1967 Theory of recursive functions and effective computability, McGraw-Hill, New York.

J. C. Shepherdson

1960 Representability of recursively enumerable sets in formal theories, Arch. Math. Logik Grundlagenforsch. 5 119-127.

C. Smoryński

A Calculating self-referential statements. II: non-explicit calculations (to appear).

429 SOUTH WARWICK, WeSTMONT, ILLINOIS 60559 